Journal of Astronomical Instrumentation, Vol. 6, No. 2 (2017) 1740008 (13 pages)

(C) The Author(s)

DOI: $10.1142 /$ S2251171717400086

\title{
POLOCALC: A Novel Method to Measure the Absolute Polarization Orientation of the Cosmic Microwave Background
}

\author{
Federico Nati ${ }^{1,6}$, Mark J. Devlin ${ }^{1}$, Martina Gerbino ${ }^{2}$, Bradley R. Johnson ${ }^{3}$, \\ Brian Keating ${ }^{4}$, Luca Pagano ${ }^{5}$ and Grant Teply ${ }^{4}$ \\ ${ }^{1}$ Department of Physics and Astronomy \\ University of Pennsylvania, Philadelphia, 19104 PA, USA \\ ${ }^{2}$ The Oskar Klein Centre for Cosmoparticle Physics \\ Department of Physics, Stockholm University \\ SE-106 91 Stockholm, Sweden \\ ${ }^{3}$ Department of Physics \\ Columbia University, New York, NY 10027, USA \\ ${ }^{4}$ Department of Physics, University of California \\ San Diego, CA 92093-0424, USA \\ ${ }^{5}$ Institut d'Astrophysique Spatiale, CNRS, Univ. Paris-Sud \\ Université Paris-Saclay, Bât. 121, 91405 Orsay cedex, France \\ ${ }^{6}$ fnati@physics.upenn.edu
}

Received 2017 April 7; Accepted 2017 April 27; Published 2017 May 22

\begin{abstract}
We describe a novel method to measure the absolute orientation of the polarization plane of the Cosmic Microwave Background (CMB) photons with arcsecond accuracy, enabling unprecedented measurements for cosmology and fundamental physics. Existing and planned CMB polarization instruments looking for primordial B-mode signals need an independent, experimental method for systematics control on the absolute polarization orientation. The lack of such a method limits the accuracy of the detection of inflationary gravitational waves, the constraining power on the neutrino sector through measurements of gravitational lensing of the $\mathrm{CMB}$, the possibility of detecting Cosmic Birefringence (CB), and the ability to measure primordial magnetic fields. Sky signals used for calibration and direct measurements of the detector orientation cannot provide an accuracy better than $1^{\circ}$. Self-calibration methods provide better accuracy, but may be affected by foreground signals and rely heavily on model assumptions, losing constraining power on fundamental processes, like CB, Faraday Rotation and chiral gravity models. The POLarization Orientation CALibrator for Cosmology, POLOCALC, will dramatically improve instrumental accuracy by means of an artificial calibration source flying on high-altitude balloons and aerial drones. Polarization angle calibration requires observation of a well-characterized distant source at high elevation angles. A balloon-borne calibrator will provide a source in the far field of larger telescopes, while an aerial drone can be used for tests and smaller polarimeters. POLOCALC will also allow a unique method to measure the telescopes' polarized beam. Even a two-hour balloon flight will allow enough time to perform polarization angle calibration and polarized beam function measurements. The source will make use of both narrow and broadband microwave emitters between $40 \mathrm{GHz}$ and $150 \mathrm{GHz}$ coupled to precise polarizing filters. The orientation of the source polarization plane will be registered to absolute celestial coordinates by star cameras and gyroscopes with arcsecond accuracy. This project can become a rung in the calibration ladder for the field: any existing or future CMB polarization experiment observing our novel polarization calibrator will enable measurements of the polarization angle for each detector with respect to absolute sky coordinates.
\end{abstract}

Keywords: Cosmic microwave background, polarization, systematics, B-modes, high-altitude balloons.

\footnotetext{
${ }^{6}$ Corresponding author.

This is an Open Access article published by World Scientific Publishing Company. It is distributed under the terms of the Creative Commons Attribution 4.0 (CC-BY) License. Further distribution of this work is permitted, provided the original work is properly cited.
} 


\section{Introduction}

Existing and planned Cosmic Microwave Background (CMB) polarization instruments need an independent, experimental method for exquisite systematics control on the absolute polarization orientation. The lack of such a method limits the accuracy on the detection of Inflationary Gravitational Waves (IGW), the constraining power on the neutrino sector through measurements of gravitational lensing of the CMB, the possibility to detect Cosmic Birefringence (CB) that would represent a paradigm shift in fundamental physics, and the ability to measure Primordial Magnetic Fields (PMFs).

\subsection{State-of-the-art}

$\mathrm{CMB}$ intensity and polarization measurements have been an invaluable resource for testing cosmological models and fundamental physics, since processes that operated in the early Universe, or acted on the photons' way to the Earth, left very weak but distinct imprints on the uniform background. We have learned that space-time is flat, that our Universe is 13.8 billion years old and its energy content is dominated by cold dark matter and dark energy (Planck Collaboration, 2014a, 2016a). The polarization anisotropy patterns are a combination of even-parity (E) and odd-parity (B) modes. According to the standard cosmological model, first-order density (scalar) perturbations at the last scattering surface produced both intensity and E-mode polarization anisotropies, while B-modes require tensor perturbations with parity-odd components. Recent E-modes polarization measurements are consistent with the standard cosmological model (Planck Collaboration, 2014b, 2016b; Louis et al., 2016). Primordial B-mode signals have never been detected. They are fainter and can be easily contaminated, yet they may reveal crucial information on our Universe. Many major questions may find their answers in the subtle B-mode signals, like: did inflation really happen? What is the signal level of primordial gravitational waves predicted by inflation? How many neutrino species are there and what is their mass? Were magnetic fields already present in the early Universe? Where do magnetic fields in galaxies and galaxy clusters come from? Accurate measurements of B-modes can also reveal (or place limits on) CB, a revolutionary departure from the Standard Model that allows to probe the validity of fundamental symmetries, and to investigate the nature of Dark Energy and test extensions of the General Relativity. To move forward, current and future $\mathrm{CMB}$ polarization experiments are aiming at an unprecedented level of sensitivity, therefore systematic effects that until today were less important than statistical uncertainties are becoming the most significant limitation. An apparent polarization rotation produces a power leakage from E-mode spectrum into B-mode signal that results in a systematic signal in the B-modes spectrum and in the TB and EB cross-spectra encoding the correlation of temperature $(\mathrm{T})$ and E-mode signals with B-modes. As a road map for ground experiments and future satellite missions looking for primordial B-modes has been outlined (CMB-S4 collaboration, 2016; Di Valentino et al., 2016), it is time to overcome the existing experimental methods to calibrate the polarization angle that only allow a total accuracy around $1^{\circ}$ (Abitbol et al., 2016; Planck Collaboration, 2016c; de Bernardis \& Masi, 2016). Such a systematic noise has in fact emerged above existing and forecasted sensitivity levels, as we will show later.

\subsection{Astrophysical and instrumental origins of $B$-modes}

Origins of B-modes can be either primordial or more recent and they can be either astronomical or instrumental. Several effects also produce nonvanishing $\mathrm{TB}$ and $\mathrm{EB}$ cross-angular cross-power spectra, including not only instrumental systematics, but also genuine physical phenomena affecting the orientation of the polarization plane of the CMB photons and galactic foregrounds, limiting the accuracy of self-calibration methods which rely on the assumption that the origin of $\mathrm{TB}$ and $\mathrm{EB}$ spectra is purely instrumental (Keating et al., 2013). Spurious B-modes originated from polarization systematics convert into a bias affecting cosmological information (Shimon et al., 2008; O'Dea et al., 2007; MacTavish et al., 2008; Hu et al., 2003). In particular, a rotation of the polarization sensitive detector with respect to the reference frame used to define the cosmological Stokes parameters generates B-modes from E-modes. A miscalibration of the orientation of the polarimeter detectors is equivalent to such a coherent rotation. Any cosmic or instrumental polarization rotation converts E-mode into B-mode and vice versa, but the E-mode signals 
are significantly larger than the B-modes, so the net result is an excess on the B-mode power spectrum. An unknown bias on the polarization angle limits the accuracy of B-mode measurements, weakening the constraints on cosmological parameters, and it has a complete degeneracy with genuine physical phenomena like CB (Yadav et al., 2012; Pagano et al., 2009). While the two angular coordinates defining the CMB polarimeter's pointing direction can be calibrated with high accuracy, the third angle defining the rotation of the polarization plane along the detector's line-of-sight is much harder to refer to absolute celestial coordinates. Also, non-ideal telescope beam functions mix intensity and polarization signals, resulting in large polarization systematics if not properly detailed (Jones et al., 2007; Miller et al., 2009; O'Dea et al., 2007). In some cases, these kinds of irregularities can also produce TB or EB signals. Since the current experimental techniques are not able to significantly reduce the level of polarization angle systematics, many experiments make use of a self-calibration technique, based on the assumption that any TB or EB power in the crossspectra comes from an instrumental bias (Keating et al., 2013; Kaufman et al., 2014a). However, genuine correlation between E- and B-modes spectra from astrophysical signals limit the accuracy of self-calibration (Abitbol et al., 2016). Besides, self-calibration comes at the price of losing constraining power on fundamental phenomena that can affect the polarization orientation of the CMB photons, like CB.

One of inflation's predictions is a background of gravitational waves that propagated in the primordial Universe. IGW were tensor fluctuations that imprinted E- and B-mode polarization patterns in the CMB. We are still looking for evidence of this key prediction. Recent measurements place a limit on the ratio between the tensor to scalar perturbations produced by IGWs of $r<0.07$ (Planck Collaboration, 2016a; Ade et al., 2016). Simulations show that IGW searches targeting tensor-to-scalar ratios of $r \simeq 0.01$ must calibrate the relationship between the instrument frame and the reference frame on the sky at least with arcminute precision (Shimon et al., 2008; O'Dea et al., 2007; MacTavish et al., 2008). Subarcminute accuracy will be required for even smaller values of $r$, targeted by CMB-S4 and future satellite missions.

More recent processes strongly contaminate the subtle primordial polarization imprints. Intervening large scale structures can change the polarization properties of the CMB photons through gravitational lensing, which also encodes information on the total neutrino mass in the CMB polarization patterns. Precise lensing measurements place the most stringent limits on the neutrino sector and are necessary to separate gravitational distortions from the primordial signals (Planck Collaboration, 2014c, 2016d,e; Sherwin et al., 2016; van Engelen et al., 2016; Madhavacheril et al., 2015).

Galactic dust polarized emission dominates primordial B-mode signals at all latitudes (Planck Collaboration, 2016f) and represents the most pernicious astronomical foreground that needs to be accurately subtracted to clean the images of the early Universe. Polarized galactic foregrounds produce EE, BB, TB and EB angular power spectra. Even small non-vanishing cross-spectra limit the accuracy of self-calibration methods (Abitbol et al., 2016).

CB predicts a photon polarization plane rotation due to non-Standard Model phenomena coupling with electromagnetism. CB can break parity symmetry via a Chern-Simons coupling term (Gluscevic \& Kamionkowski, 2010) or violate the Einstein Equivalence Principle via a pseudo-scalar field interaction with light (Carroll et al., 1990). CB rotation converts E-modes into B-modes and produces TB and EB cross-spectra. CB detection would be a paradigm change in fundamental physics. $\mathrm{CMB}$ is the most distant polarized light we can observe, so it can accumulate even a very small $\mathrm{CB}$ rotation over cosmological distances (Xia et al., 2010; Yadav et al., 2012). While it is possible to isolate CB from other effects breaking the degeneracy through frequency or scale dependencies, its effect is indistinguishable from a bias on the measured polarization angle, which therefore requires an accurate independent calibration (Pagano et al., 2009; Rosset et al., 2010). Upper limits to CB from CMB polarization data have been measured (Planck Collaboration, 2016g; Ade et al., 2015; Gruppuso et al., 2016). Since the net result of CB is to increment the B-mode spectrum, if we do not take into account its effect, we introduce a positive bias for $r$. It is also important to note that if $\mathrm{CB}$ starts to accumulate during photon propagation right after recombination, before gravitational lensing mixes temperature and polarization signals, then lensed CMB spectra do not contain only primordial anisotropies, but also encode a rotation effect due to birefringence (Gubitosi et al., 2015). 
If magnetic fields were present in the primordial plasma, they affected the polarization angle of CMB photons through Faraday Rotation (FR). In other words, FR allows to measure magnetic fields in the early Universe through the detection of a rotation of the polarization orientation. Like other rotation effects, FR produces E-mode to B-mode leakage (Kosowsky \& Loeb, 1996; Pogosian et al., 2012) and, similarly to $\mathrm{CB}$, generates non-vanishing $\mathrm{TB}$ and EB spectra. While some explanations for galactic microgauss fields are provided by dynamo mechanisms, the origin of magnetic fields in large structures is still unclear. Accurate CMB measurements can place bounds on PMFs and provide explanations on their origin. Since this is a primordial effect, it mixes with the tensor B-modes caused by IGWs. FR can be distinguished from other phenomena thanks to its frequency dependence. Existing telescopes already have the sensitivity to detect mode-coupling correlations sourced by a scale-invariant PMF below 1 nanogauss (nG) or even better when the weak lensing contribution is subtracted. But, an independent and improved polarization angle calibration is required. Planck measured an upper limit for the PMFs to $4.4 \mathrm{nG}$ (Planck Collaboration, 2016h) assuming zero helicity, and a similar result was confirmed by POLARBEAR (Ade et al., 2015). By assuming maximal helicity, the FR only constraint on stochastic PMFs measured by Planck is $<1380 \mathrm{nG}$ (Planck Collaboration, 2016h), obtained using only large scale EE and $\mathrm{BB}$ modes at $70 \mathrm{GHz}$. The current limits on PMFs are usually obtained assuming zero or maximally helical field. Accurate measurements of TB and EB spectra will open the possibility of constraining the helicity of PMFs (Ballardini et al., 2015).

\section{Limitations of the Existing Calibration Methods}

The limitations of the current methods for calibrating the absolute polarization direction of the detectors are summarized in the following list:

(1) Lack of natural reference sources. Sky signals from known sources are traditionally used to calibrate the intensity of CMB experiments, but there is no analogous standard for polarized beam measurements or polarization angle calibration. The few astronomical candidates suffer from frequency dependence and time variability.
They are not visible from all observatories and are extended sources. The best option is Tau-A which allows an accuracy for the polarization orientation between $1^{\circ}$ and $0.5^{\circ}$ (Planck Collaboration, 2016i; The POLARBEAR Collaboration, 2014; Weiland et al., 2011).

(2) Given their typical dimensions, the alignment of detector arrays and cold optics with better than $1^{\circ}$ accuracy requires a positioning uncertainty smaller than $1 \mathrm{~mm}$ (de Bernardis \& Masi, 2016). Microwave detectors must be cooled down from $300 \mathrm{~K}$ to $100 \mathrm{mK}$. Differential contractions of the materials in the cryostat introduce additional misplacements larger than $1 \mathrm{~mm}$. Even with a careful design to mitigate these effects, it is hard to fully recover the accuracy, since all the parts must be mounted together at room temperature. During operations, external pressure and temperature can change, affecting the cryostat internal conditions. It is also critical to refer the detector orientation with respect to the telescope and the receiver mount once the cryostat is closed. As a result, direct polarization angle calibration is not possible with an accuracy better than $1^{\circ}$.

(3) Many experiments require the use of polarization modulator systems based on large rotating half-wave plates (HWP) (Simon et al., 2016; Thornton et al., 2016; The LSPE Collaboration, 2012). Thanks to the large filter diameter, in principle, they can calibrate the polarization angle with good accuracy. However, ideal, wideband, optically uniform and thermally stable HWPs do not exist, so they introduce uncontrolled biases degrading the accuracy (Pisano et al., 2014; Essinger-Hileman et al., 2016). Some experiments make use of reflective polarization modulation systems, but also in this case, non-ideal properties weaken the alignment accuracy (Miller et al., 2016; Chuss et al.,, 2012; Houde et al., 2001). HWP rotating mount can also be used to place a thin film polarization grid in front of the receiver and calibrate the absolute polarization orientation of the detectors (Koopman et al., 2016). However, any strategy based on optical elements placed between the mirrors and the polarimeter does not allow to measure the polarized beam systematics induced by the warm optics.

(4) The characterization of the polarized beams requires a calibrated, polarization-pure source 
placed in the far field of the telescope. Even the best candidates among the astronomical sources that could be used for this scope, like Tau-A (Planck Collaboration, 2016i; The POLARBEAR Collaboration, 2014; Weiland et al., 2011), are not point-like sources, and their frequency spectrum within the sensitive bands of the polarimeters is not precisely known.

(5) The standard cosmological model predicts that in the early Universe, the odd-parity and the even parity signals should be completely unrelated. The angular power spectrum is commonly used to study the statistical properties of the CMB anisotropies. It represents the variance of the signal fluctuations in temperature (TT) and polarization (EE, BB) for different angular scales. The cross-spectra indicated as TE, TB and EB, measure the correlation across temperature and polarization modes. The standard cosmological model predicts that TB and EB identically vanish, which means that the oddparity signal (B) should be completely unrelated to intensity $(\mathrm{T})$ and even-parity signals $(\mathrm{E})$ in the early Universe. This prediction can be used to calibrate CMB polarimeters through a self-calibration method (Keating et al., 2013; Kaufman et al., 2014a), at the expense of losing detection capability on genuine physical quantities. This method is particularly effective for high resolution and/or high sky coverage experiments. However, the initial assumption is not true in the presence of phenomena that produce non-vanishing TB and EB. In these cases, self-calibration loses accuracy and introduces biases on cosmological parameters (Abitbol et al., 2016). Besides, this method destroys the possibility to measure or to place limits on phenomena that generate TB and EB spectra, like $\mathrm{CB}, \mathrm{FR}$ and chiral gravity models (Kaufman et al., 2014b; Gerbino et al., 2016). TB and EB correlations can also be introduced by polarized galactic foregrounds and instrumental systematics.

\section{A Novel Method: POLOCALC}

POLOCALC will overcome current limitations by implementing a novel approach to calibrate the polarization angle and the beam pattern of the CMB polarimeters with an accuracy between $0.01^{\circ}$ and $0.001^{\circ}$. Such a method will fully enable the constraining power of the CMB data set with no change to existing or planned instruments.

\subsection{Objectives}

POLOCALC objectives are divided as follows:

(i) Enabling unprecedented accuracy on the measurement of IGW and the tensor-to-scalar ratio through a novel calibration method insensitive to polarized foreground emission;

(ii) Controlling systematics on CMB gravitational lensing signals, improving the accuracy on gravitational potential measurements and the resulting constraints on the neutrino sector;

(iii) Revealing (or constraining) $\mathrm{CB}$ and physics beyond the Standard Model that can violate fundamental symmetries;

(iv) Measuring PMFs through FR, anchoring inflationary models and providing evidence of the seeds that originated magnetic fields in large structures.

POLOCALC will utilize two celestial coordinates (from the accurate star camera pointing direction) to determine the third angle defining the rotation of the polarization plane along the detectors' lineof-sight. The camera pointing direction is aligned to a known polarized source operating from a balloon-borne payload in the far field of ground telescopes. POLOCALC will fly in the Atacama desert (Chile), within view of the Simons Observatory which includes the ACT and Simons Array telescopes (Simons Observatory Collaboration, 2016). The telescopes' detector orientation is then measured by observing the calibration source signal. CMB polarimeters will observe our artificial, wellcharacterized linear polarization source in the far field of the fully integrated instruments operating at cryogenic temperatures. The calibration sources will be visible at high elevation angles, far from Earth signal contamination. Requirements of such long distances, small zenith angles and high angular accuracy make this measurement extremely challenging and never attempted before. Flying in the Atacama region will maximize the impact of POLOCALC: any existing or future project in the area, including ALMA and CLASS, will be able to use this novel polarization calibrator which will enable measurements of the polarization angle for each detector with respect to absolute sky coordinates. 


\subsection{Methodology}

POLOCALC will implement a novel calibration method to measure the polarization angle and the telescope beam patterns making use of precisely characterized, linearly polarized microwave sources. These artificial calibrators will match the sensitive frequency bands of the CMB polarimeters and will operate from a balloon-borne payload, illuminating the ground telescopes from far-field distances, appearing as distant sources for the polarization sensitive detectors of the instruments. POLOCALC will be tested on the ground and on a flying drone before being operated from a high-altitude balloon, as shown in Fig. 1. This will allow us to test the method with increasing gradations of risk and performances, comparing far- and near-field results.

The calibration source must be placed at large distances and observed by the telescopes at low zenith angles (high elevation angles). In the case of ACT, POLARBEAR/Simons Array and Simons Observatory telescopes, the far-field distances range between $5 \mathrm{~km}$ and $36 \mathrm{~km}$ for the sensitive bands centered between $40 \mathrm{GHz}$ and $150 \mathrm{GHz}$. For smaller telescopes, like CLASS, the far-field distances are of the order of $100 \mathrm{~m}$. In a first test, we will place the sources on nearby mountains in sight of the telescopes, for example, close to the TAO telescope on the summit of Cerro Chajnantor. For small and medium size instruments, ground sources will provide far-field measurements. While this strategy already crosses the accuracy thresholds of current methods, it comes with some limitations. To observe a ground-based calibration source, the telescopes must point almost horizontally, so the polarimeters will receive a huge loading background from the thermal emission of the Earth and the warm air mass. These signals will saturate any typical $\mathrm{CMB}$ detector system which is optimized to observe cold sky signals at temperatures lower than $10 \mathrm{~K}$. Even if there are methods to attenuate the loading on the detectors, they introduce additional systematic errors affecting the calibration accuracy. Besides, there are telescopes, like ACTPol, that cannot point at low elevation angles and require at least $36 \mathrm{~km}$ of far-field distance at $150 \mathrm{GHz}$. A second test measurement will overcome the ground-based limitations by mounting the calibration system on a drone platform flying at $100 \mathrm{~m}$ above the telescopes. The drone is coupled with a stabilization system that will reduce payload oscillations and will constantly point the source towards the experiments. The source will have a large beam, so pointing accuracy is not as important as attitude determination, which will be provided by a separate system. For small aperture telescopes like CLASS, the drone already provides far-field calibration. For larger mirrors, a near-field measurement will also provide arcminute calibration when paired with telescope optical models to simulate beam propagation (Koopman et al., 2016) and extrapolate the results for pointlike sources.

The balloon-borne configuration will fully overcome all existing limitations enabling both far-field and high elevation (small zenith angles)

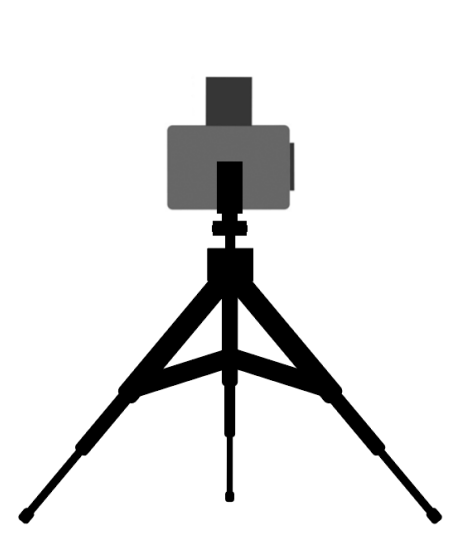

(1)

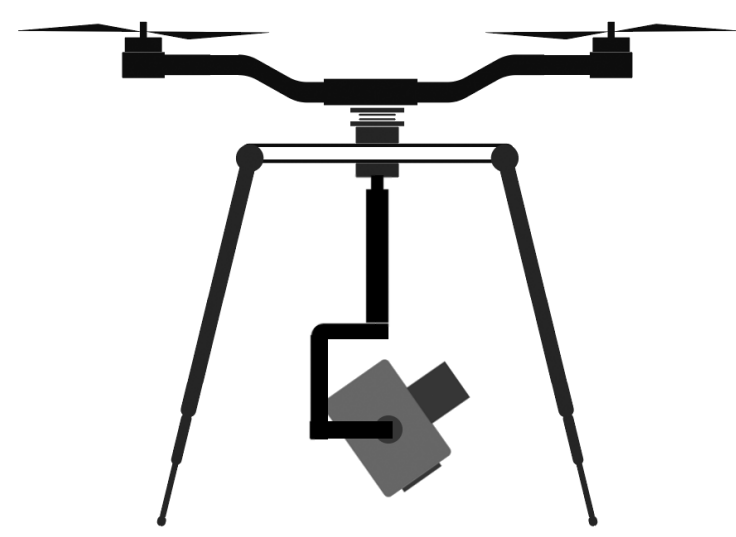

(2)

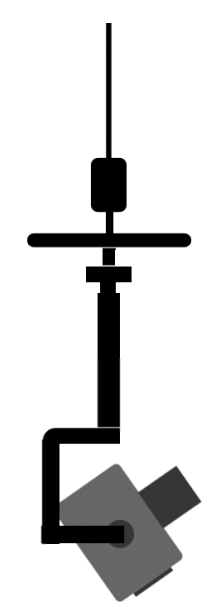

(3)

Fig. 1. Three different configurations for POLOCALC (starting from the left): (1) from a ground tripod, (2) from a flying drone and (3) from a high-altitude balloon. 
measurements for medium and large telescopes. POLOCALC will make use of high-altitude balloons flying in sight of the experiments at around $30 \mathrm{~km}$ of altitude.

POLOCALC will provide sources with polarization orientation registered in absolute celestial coordinates with an accuracy between $0.01^{\circ}$ and $0.001^{\circ}$. We will measure the detectors' absolute orientation with unprecedented accuracy and without cosmological model assumptions. The systematics control will match the sensitivity levels expected for CMB-S4. The absolute orientation of the polarized source will be provided with arcsecond accuracy thanks to a state-of-the-art attitude control system (ACS) based on star cameras, gyroscopes, and other orientation sensors like encoders, clinometers, and magnetometers. A precisely machined frame will firmly hold together the ACS star camera with the microwave source and filter, with the respective pointing axis separated by $90^{\circ}$, as shown in Fig. 2. In this way, when the microwave source points towards the telescope, the star camera will look at the sky. Our software will recognize the stars' positions captured in the images and determine the absolute orientation of the source. The pointing direction of the star camera is aligned and parallel to the polarization direction of the microwave source output with arcsecond accuracy. In other words, the camera pointing solution also provides the orientation of the microwave source linearly polarized light. This novel approach makes use of the high accuracy on the determination of the two celestial angular coordinates of the star camera pointing solution to determine the absolute orientation of the source polarized light with the same exquisite arcsecond accuracy. While similar ground calibrations have already been performed (Kaufman et al., 2014a), a sky-referenced platform like POLOCALC has never been used before. POLOCALC will make an unconventional use of professional stabilization systems for drone video recording and handheld cameras to stabilize the pointing of the calibration source even on balloons. POLOCALC's accuracy will be limited by the uncertainty of the star camera solutions, the mechanical alignment precision of the linear polarization filter direction of the source with the ACS, and the thermal stability and uniformity of the payload. State-of-the-art ACS can typically provide an absolute orientation determination with an accuracy between $0.01^{\circ}$ and $0.001^{\circ}$ (Gandilo et al., 2014; Chapman et al., 2014). The same accuracy range can be achieved for the assembly of the camera and the wire-grid, making use of precise rotary stages, microscopes, and accurate metrology systems. We will implement a thermal control providing temperature stability between $2 \mathrm{~K}$ and $0.2 \mathrm{~K}$, corresponding to an angular misalignment on the order of $0.01^{\circ}$ and $0.001^{\circ}$, respectively.

It is important to highlight that we will transfer the calibration obtained with POLOCALC among different detectors and telescopes by observing the same sky signals, reducing the risk of calibration mismatch between detectors. This is also useful both to compare the accuracy of different instruments and to have the telescopes with ground screens limiting their view to benefit from ground calibration. For example, POLARBEAR/Simons Array telescopes can already make use of the ground measurements, and the results of the calibration can be used for systematics control on the ACTPol instruments via shared observation of celestial sources or CMB fields. Future telescopes like the Simons Observatory will also be able to point at zero elevation.

POLOCALC's sky-referenced, balloon-borne polarization orientation calibrators will overcome, one by one, the five main limitations of the current calibration methods listed above, enabling

(1) Accurate calibration of the polarization angle for ground $\mathrm{CMB}$ polarization experiments thanks to a precisely characterized distant artificial source;

(2) Calibration of fully integrated and cold receivers, avoiding compensation for mechanical misalignments and differential thermal contractions between laboratory and operating conditions;

(3) Systematics control over thermal, optical or mechanical irregularities of beam filling HWP or polarization filters. Calibration of the full optical chain, including warm mirrors;

(4) Near- and far-field measurements of the telescope beam patterns. Calibrating the detectors' polarization angle and their polarized beams are two different tasks, but for both of them a distant artificial source is invaluable;

(5) Calibration without assumptions on the primordial coupling of intensity and polarization modes, as opposed to the self-calibration methods, thus preventing the induced biases on the cosmological parameters and enabling CB measurements. 


\subsection{Implementation}

\subsubsection{Sources}

POLOCALC will make use of multiple sources with frequency bands centered between $90 \mathrm{GHz}$ and $150 \mathrm{GHz}$. We will utilize commercially available microwave oscillators, horns and filters, but we will also develop and build custom emitters. The sources will optimally match the brightness and frequency requirements of the polarimeters. Frequency multipliers and filters will provide the desired output frequency band. Depending on the mirror size, source distance, detector noise and frequency band, the final output power will be adjusted using the formulas in Lamarre (1986). As an example, for sources at $90 \mathrm{GHz}$ and $150 \mathrm{GHz}$ placed $5 \mathrm{~km}$ away from the instrument, the required power is $500 \mathrm{pW}$ and $30 \mathrm{pW}$, respectively, which means attenuation factors of $50 / 60 \mathrm{~dB}$ for a $50 \mathrm{~mW}$ Gunn oscillator. We will couple the source to rectangular waveguides and horns to provide a linearly polarized microwave light with $-30 \mathrm{~dB}$ of cross-polarization level. A polarization filter will be used to further clean or adjust the reference signal. We will use precise photolithographed wire-grid polarization filters. The inside of the box containing the source will be covered with radio absorbing material and we will also consider the use of mesh filters to mitigate reflections between the filter and the horn. We will precisely align and measure the polarization direction with arcsecond accuracy with respect to the ACS camera. The frame holding together all the parts requires a machining precision of $0.05 \mathrm{~mm}$ or better. We will precisely characterize the microwave source in laboratory before deployment. The beam pattern of the source will also be precisely measured using rotary stages, diode sensors and lock-in amplifiers. During the operations in the field, the microwave source power will be modulated for optimal signal recovery using a lock-in demodulation technique, while on the telescope side, we will also take advantage of the modulation systems such as a rotating HWP to change the polarization orientation.

\subsection{2. $A C S$}

The ACS is a custom composition of different subsystems with the scope of measuring and controlling latitude, longitude, altitude and the three-axis absolute orientation of the polarized emitter. It is composed of a central processing unit, optical sensors, attitude sensors, digital encoders and motors.

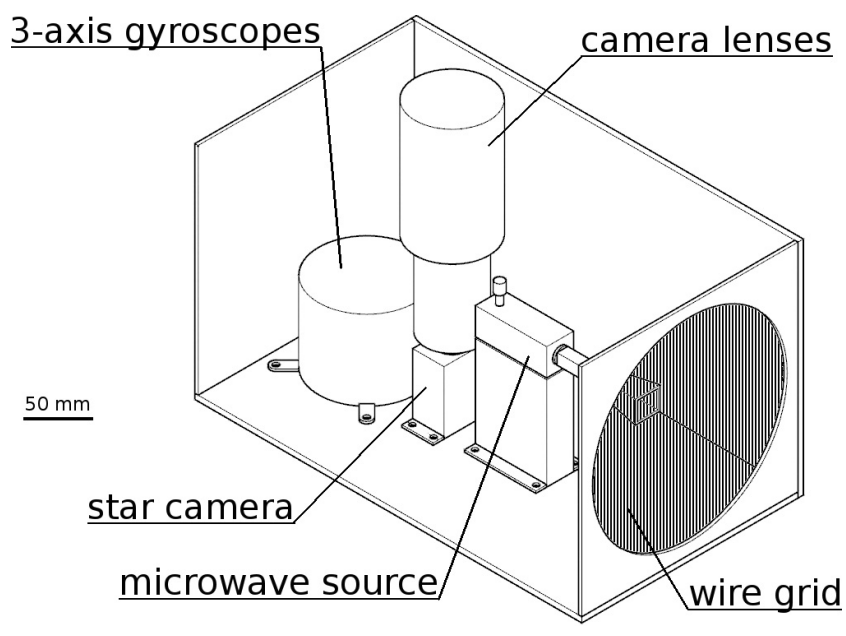

Fig. 2. A schematic representation of the essential elements of POLOCALC. From right to left: the linear polarization filter, the microwave source pointing towards the telescopes, behind it the star camera looking at the sky (oriented at $90^{\circ}$ with respect to the source direction), and the three-axis high-precision gyroscopes.

POLOCALC will use the same ACS on the ground, on the flying drone and on the high-altitude balloon. We will utilize a state-of-the-art professional camera stabilization system which makes use of gimbals and motors to ensure high rejections of the undesired trembling of the payload pointing. We will stabilize the microwave source and the star camera with this system. While it is designed for drones and handheld video recording, for the first time, we will adapt a camera stabilization system on a small balloon payload to point steadily towards the telescope. Units like the FreeFly systems weight around $2 \mathrm{~kg}$, have a battery life of several hours, and can be remote controlled or programmed in target mode. We will protect and thermally isolate the stabilization system's critical parts and test them in a environmental chamber simulating the stratospheric environment. We will also develop and test an independent pointing motor to stabilize the azimuth motion on the balloon payload. The balloon moment of inertia is small, so reaction wheels will provide enough moment of inertia and torque for azimuth movements. Due to the short operating time at float, it is unlikely that the reaction wheel will saturate due to friction and balloon rotation, but we will consider the use of a small azimuth motorized pivot to prevent this issue. Other custom solutions to help to recover from reaction-wheel saturation can be explored if needed. Attitude stability is a requirement for the star camera to take still pictures of the sky, and for the microwave 
source in order to reduce the effect of instrument time constants. POLOCALC will take advantage of improved and miniaturized technology using a small, light, high sensitivity, high dynamic range, low noise star camera coupled to professional lenses and a filter at $530 \mathrm{~nm}$ to enhance star signals over atmospheric noise. The static pointing of POLOCALC allows frequent image acquisitions from the star camera. The star-field images will be stored on a Solid State Drive which is light, low consumption and does not require pressurization. Three-axis laser gyroscopes will be integrated over time for fast and precise attitude reconstruction. Further attitude sensors like clinometers, and magnetometers will contribute to the alignment solution. We will use GPS time to synchronize the pictures and the pointing solutions with the raw telescope data. During the flights, temperature gradients across the payload produce misalignments between the filter and the camera boresight, and even uniform temperature variations of the payload cause misalignments due to different materials of the aluminum holding frame and of the dielectric substrate of the polarization filter. Variations of $2 \mathrm{~K}$ and $0.2 \mathrm{~K}$ correspond to misalignments of the order of $0.01^{\circ}$ and $0.001^{\circ}$, respectively. We will then utilize an active thermal control providing the required temperature stability and uniformity within these ranges. We will also insulate the vessel with foam and aluminized mylar layers.

\subsubsection{Flight operations}

A high-altitude balloon with a diameter of $\sim 3 \mathrm{~m}$ and a weight of $\sim 2 \mathrm{~kg}$ can be hand-launched and take the payload to the stratosphere in around an hour. A typical two-hour flight will terminate at around $30 \mathrm{~km}$ from the launching site. We will choose the launching site so that the ground telescopes can observe the source at float at an elevation angle larger than $45^{\circ}$. Line-of-sight and Iridium-based telemetry systems will relay the GPS position of the source to the ground. The telescope pointing systems will utilize this information to track the source and perform beam maps. Total payload weight will be limited to less than $10 \mathrm{~kg}$ by the drone and the balloon restrictions. In January 2017, an aerial drone used to film the telescopes at the Cerro Toco site showed feasibility for flying a stabilized camera at around $100 \mathrm{~m}$ above the experiments. We will adapt a similar drone to our project. Stratospheric balloon flights will allow operations of the calibrators from $30 \mathrm{~km}$ of altitude or more. POLOCALC balloons will fly above the Atacama Desert in Chile, allowing visibility from all ground telescopes in the area. We will select rugged hardware and protect all delicate parts for use in the harsh conditions during deployment. In the stratosphere, the operating pressure is only a few mbar, so we will test all the parts in a vacuum chamber in the laboratory. We will design the temperature control system to keep all the parts in their working range. To allow optimal observations, the balloon will be launched during the night, but daytime flights are also possible in case of restrictive wind conditions. Multiple pointing strategies will be available thanks to the movements allowed by the ACS and the stabilization system. Depending on the wind conditions, the payload will stay in sight of the telescopes for few hours approximately. The lock-in technique and the high signal-to-noise ratio from the source allow short integration times, so such a flight duration is enough to calibrate the instruments and generate beam maps. POLOCALC total consumption will be less than $25 \mathrm{~W}$. For ground use, either a lead battery pack or lithium battery pack is suitable, while for the flight, we will make use of high capacity lithium battery packs, that can easily provide $150-200 \mathrm{Wh} / \mathrm{kg}$. The balloon payload only requires a light, low consumption telemetry to relay tracking data for the ground antennas' auto-pointing and to allow access for all the telescopes that need to track the source. Drone flights will also be used to test telescopes tracking systems. Airliners and public risk are not of significant concern due to the operating area.

\section{Scientific Impact}

An anisotropic rotation of the polarization angle produces a signal leak from E-mode or temperature spectra to B-mode signals. It also produces a correlation between these signals represented by the TB and EB cross-spectra. The following simulations show the effect of a common miscalibration angle, i.e. shared by all detectors. In Fig. 3, we show the systematic errors in the $\mathrm{BB}, \mathrm{TB}$ and $\mathrm{EB}$ power spectra produced by polarization rotation. The red curves show the spurious signal produced by $1^{\circ}$ of rotation, which is approximately the current error level set by the systematic uncertainty in available calibration sources. With an improved calibration accuracy between $0.01^{\circ}$ and $0.001^{\circ}$, POLOCALC 

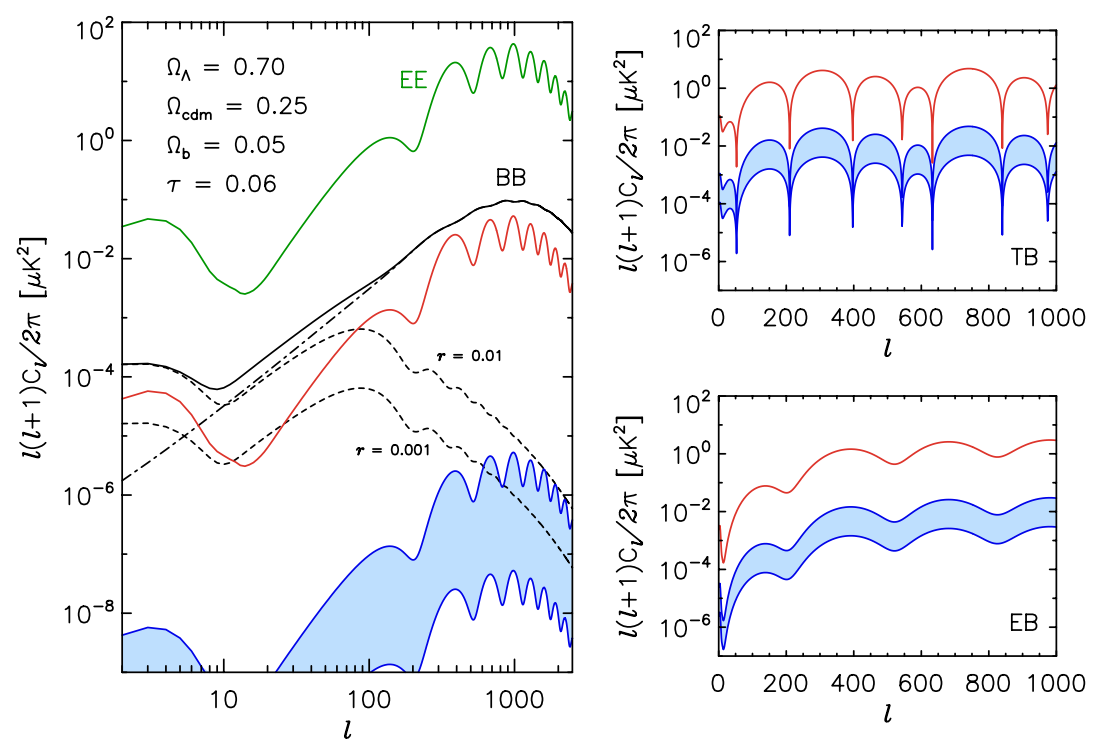

Fig. 3. Systematic errors in the BB, TB and EB power spectra produced by polarization rotation. The red curves show the spurious signal produced by $1^{\circ}$ of rotation, which is approximately the current error level set by the systematic uncertainty in available calibration sources. With an improved calibration accuracy between $0.01^{\circ}$ and $0.001^{\circ}$, POLOCALC will allow any rotation errors to be suppressed to the blue region in all plots. This suppressed error level is negligible for current experiments targeting $r \simeq 0.01$ and future experiments like CMB-S4 that will target $r \simeq 0.001$. Primordial B-mode signals (dashed), the gravitational lensing B-mode signal (dash-dot), and the EE signal (green) are plotted for reference in the left panel.

will allow any rotation errors to be suppressed to the blue region in all plots. This suppressed error level is negligible for current experiments targeting $r=0.01$ and future experiments like CMB-S4 that will target $r=0.001$. Primordial B-mode signals (dashed), the gravitational lensing B-mode signal (dash-dot), and the EE signal (green) are plotted for reference in the left panel. In the following sections, we present the expected scientific impact of POLOCALC, which can be preliminarily evaluated by simulating CMB data with noise properties that match those expected from current (Advanced ACTPol) and near future (CMB-S3) experimental configurations. The posterior distributions are then obtained by performing a Markov Chain Monte Carlo analysis employing the public COSMOMC package (Lewis et al., 2002) and an exact likelihood approach (Perotto et al., 2006).

\section{1. $I G W$}

A miscalibration of $0.5^{\circ}$ in the polarization orientation translates into a spurious B-mode signal corresponding to a tensor-to-scalar ratio of $r \simeq 0.01$ (Abitbol et al., 2016), affecting the sensitivity range of existing and planned experiments. POLOCALC will calibrate the polarization angle with arcsecond accuracy. As a consequence, the uncertainty on the value of $r$ will be limited by the sensitivity of the experiment. A simulated result is shown in Fig. 4, for an ACT-like and a CMB-S3 experiment, where the red curve represents a false bias signal introduced by a miscalibration of $1^{\circ}$ (i.e. the current accuracy) in the orientation of the detectors. The bias starts to emerge above the statistical uncertainty for ACTPol sensitivity (the image on the left). For CMB-S3 (the image on the right), the new generation of ground experiments, the bias is well above the sensitivity and it dramatically affects the measurement of $r$. If the same experiment benefits from the POLOCALC calibration, gaining an accuracy between $0.01^{\circ}$ and $0.001^{\circ}$, the bias can be recovered as represented by the blue region. For CMB-S4, the gap between accuracy and sensitivity will increase, so the effect on cosmological parameter estimations will be even more important.

\subsection{Lensing and foregrounds}

While CMB photons leave the Last Scattering Surface shortly after the Big Bang, matter distribution introduces gravitational lensing at later times. However, on the one hand, E-modes could be converted into B-modes through a polarization plane rotation before lensing happens. On the other hand, non-vanishing TB and EB spectra from local 

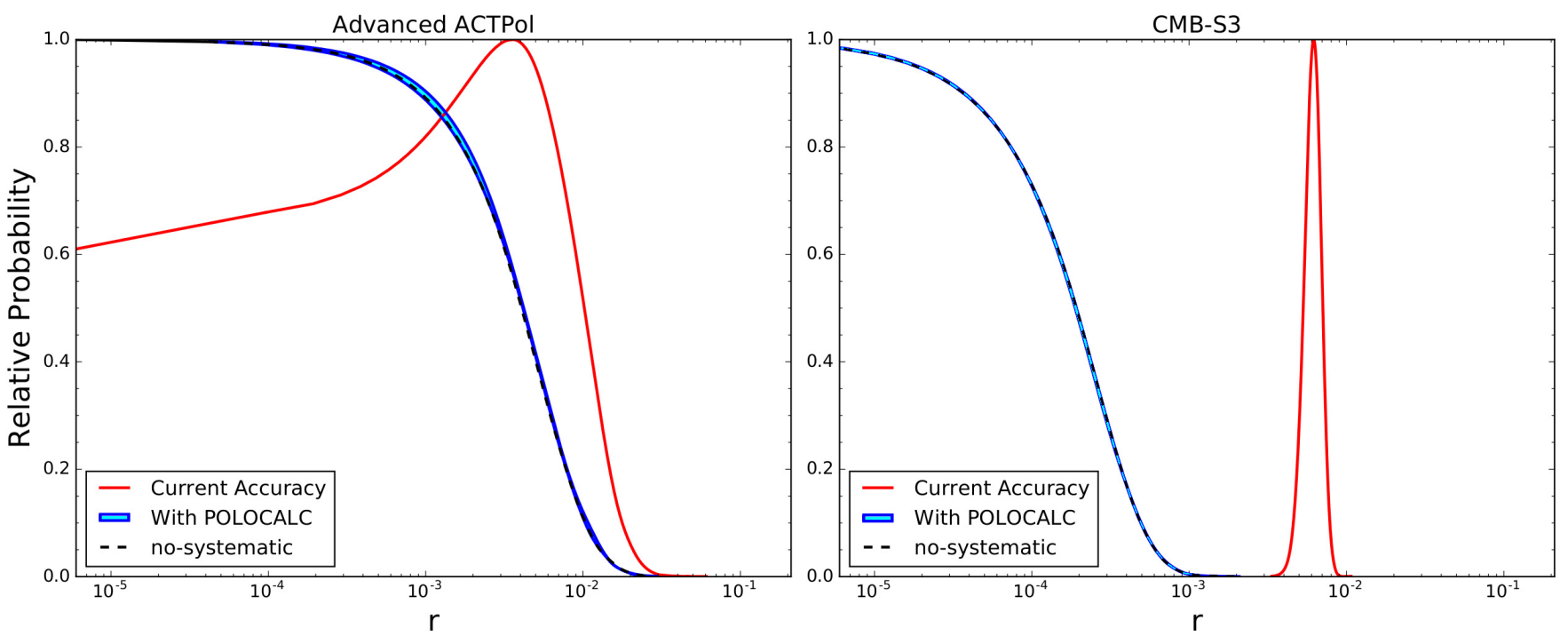

Fig. 4. Assuming no gravitational waves, the red curve in these simulations represents a false detection of $r$ caused by the polarization angle miscalibration of $1^{\circ}$. With a calibration accuracy between $0.01^{\circ}$ and $0.001^{\circ}$ represented by the blue region, POLOCALC recovers the fiducial value of $r=0$ (the black dashed curve). The uncertainty on the value of $r$ will be then limited by the sensitivity of the experiment. The false bias already starts to emerge above the statistical uncertainty for the ACTPol sensitivity, while for CMB-S3, it dramatically affects the measurement of the tensor-to-scalar ratio. For CMB-4, the gap is going to increment.

Galactic foregrounds can be present in the observations. In these cases, lensing measurements will suffer from an uncontrolled bias. This would affect both the lensing potential reconstruction and the delensing efficiency. POLOCALC calibration does not require assumptions on the polarization rotation history or on the correlations between the spectra. Therefore, POLOCALC will enable gravitational lensing potential measurements limited by statistical uncertainties. The sum of neutrino masses is one of the most relevant parameters encoded in CMB lensing signals. In Fig. 5, the black dashed curve represents the probability distribution of the sum of the neutrino masses as it would be constrained by an experiment as sensitive as ACT, if systematic effects (such as the error in the orientation of the detectors) are perfectly under control. The red curve clearly shows the bias induced by a
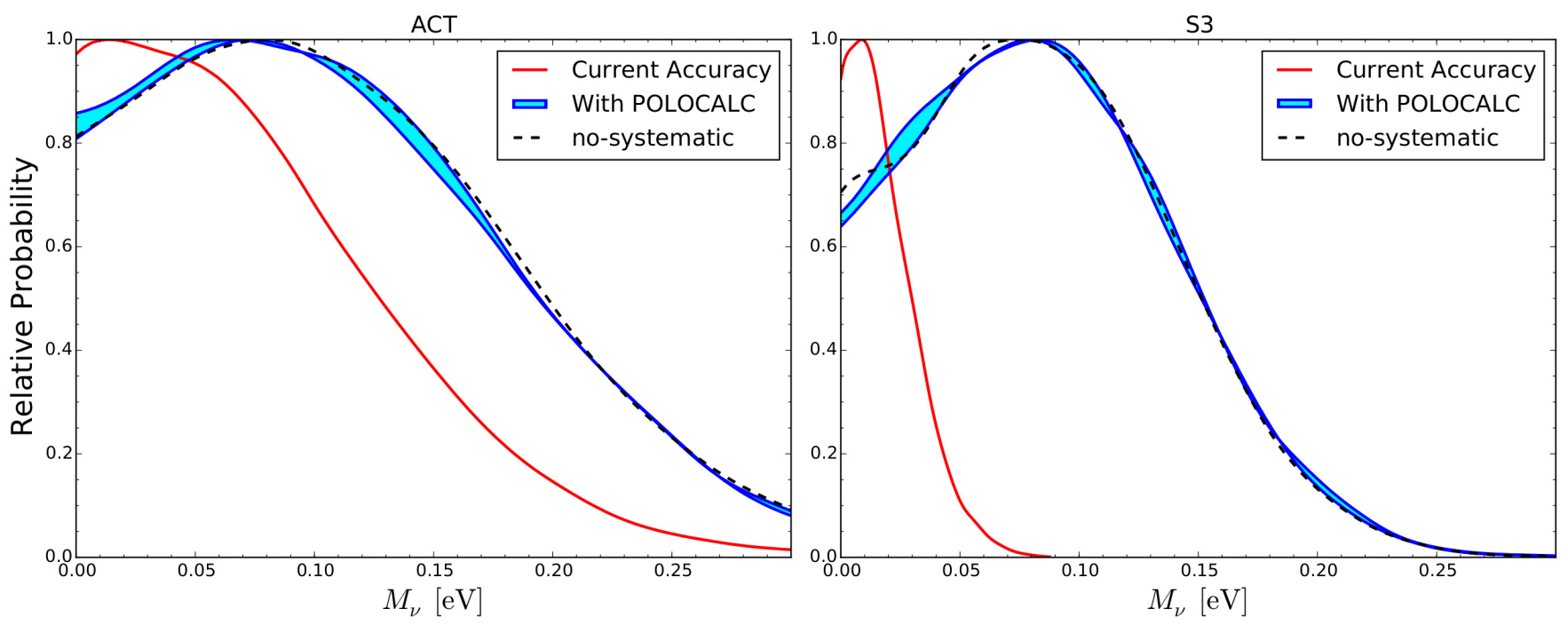

Fig. 5. The red curve in these simulations represents a wrong estimation of the sum of neutrino masses (in this model, assumed as $0.06 \mathrm{eV}$ ) caused by a polarization angle miscalibration of $1^{\circ}$ for AdvACTPol-like and CMB-S3 experiments. With a calibration accuracy between $0.01^{\circ}$ and $0.001^{\circ}$ represented by the blue region, POLOCALC recovers the fiducial value (the black dashed curve). Such a gap is going to be more relevant for CMB-S3 and CMB-S4. 
miscalibration of $1^{\circ}$ (i.e. the current accuracy) in the orientation of the detectors. If the same experiment benefits from the POLOCALC calibration, gaining an accuracy between $0.01^{\circ}$ and $0.001^{\circ}$, the bias can be recovered as represented by the blue region.

\subsection{Cosmic birefringence}

Detecting a Lorentz- and parity-violating process like CB would be a paradigm shift in physics. The outcome of a similar discovery would be revolutionary, and its consequences would deeply affect our understanding of fundamental processes and the description of our Universe. Since a polarization angle miscalibration is indistinguishable from CB, self-calibration methods come at the price of losing sensitivity to CB. POLOCALC calibration will enable measurements of the $\mathrm{CB}$ rotation angle, $\alpha_{\mathrm{CB}}$, providing an independent, experimental calibration method with an unprecedented accuracy on the absolute polarization orientation between $0.01^{\circ}$ and $0.001^{\circ}$, reaching the limit of statistical uncertainties required by CMB-S4.

\subsection{PMFs}

While CB is a departure from the Standard Model, FR is a well-known physical effect that may be used to measure the intensity of PMFs in the early Universe and select cosmological models. PMFs could also explain the presence of magnetic fields and their evolution in the Universe. The FR angle affecting the polarization plane of CMB photons is directly proportional to the magnetic field intensity. POLOCALC will improve the accuracy on the polarization orientation from $1^{\circ}$ to better than $0.01^{\circ}$, therefore scaling by two orders of magnitude the sensitivity to PMFs through FR with respect to current possibilities.

\subsection{Additional advantages}

From an experimental point of view, the importance of using a fully characterized source to illuminate the telescopes goes even beyond the scope of the polarization angle calibration and beam functions characterization. CMB experiments make use of technologies with frequency-dependent performances within the broad sensitivity bands of the polarimeters. For example, technologies like achromatic HWPs (Pisano et al., 2014) and sinuous antennas have frequency-dependent performances.
While the frequency spectrum of a celestial source is not precise enough to recover these in-band features, both POLOCALC narrowband and broadband sources have the advantage of being fully characterized in laboratory, before deployment. Therefore, they can be used to study finer in-band instrumental features during the observational campaigns. POLOCALC clean and narrowband signals can be used to compare the polarimeter transfer functions during operations with the lab-measured ones. POLOCALC can also be used to measure the modulation efficiency and polarimeter axis angle curves for an achromatic HWP polarimeter, or the polarization angle dependency of a broadband sinuous antenna. Another important advantage of POLOCALC is to provide a calibration source to inter-calibrate each polarization sensitive detector of the same instrument, and even across different telescopes.

\subsection{Advantages over a CubeSat}

While a low Earth orbit small satellite like a CubeSat could also provide a distant calibration source (Johnson et al., 2015), an orbital mission has the following disadvantages with respect to the POLOCALC balloon-borne calibrators:

(1) Assuming a polar orbit with an altitude of $500 \mathrm{~km}$, a CubeSat-based instrument would be visible from the telescopes in the Atacama Desert for only about $2 \mathrm{~min}$ in a given orbit and for only a few times each week. The satellite would need to be tracked by the telescopes while it crosses the sky, but the angular speed of the satellite can at times exceed $0.6^{\circ} / \mathrm{s}$, which is a common upper limit for telescope mounts. Therefore, making beam maps will be challenging.

(2) CubeSats pose much stronger limitations in size, weight and power. These constraints limit the calibration source technologies that can be used. Also, the broadcast frequency bands are restricted by international regulations, so the available frequencies may not optimally match the spectral bands in the polarimetric receivers.

(3) Finally, a satellite is a high risk enterprise, it can be more expensive, and it would not be available immediately.

\section{Conclusion}

POLOCALC has the potential to become a rung in the calibration ladder for existing or future $\mathrm{CMB}$ 
experiments observing our novel polarization calibrator. This novel method will enable measurements of the polarization angle for each detector with respect to absolute sky coordinates with unprecedented accuracy. This project will produce the first independently calibrated measurement of the polarization angles of the CMB light and its contaminants allowing $\mathrm{CMB}$ polarization experiments to fully mine the cosmic sky.

\section{References}

Abitbol, M. H. et al. [2016] MNRAS 457(2), 1796.

Ade, P. A. R. et al. [2015] Phys. Rev. D 92, 123509.

Ade, P. A. R. et al. [2016] Phys. Rev. Lett. 116, 031302.

Ballardini, M., Finelli, F. \& Paoletti, D. [2015] J. Cosmol. Astropart. Phys. 1510(10), 031.

Carroll, S. M. et al. [1990] Phys. Rev. D 41, 1231.

Chapman, D. et al. [2014] Proc. SPIE 9152, 915212.

Chuss, D. T. et al. [2012] Appl. Opt. 51(2), 197.

CMB-S4 Collaboration [2016] CMB-S4 Science Book, 1st edition, arXiv: 1610.02743 .

de Bernardis, P. \& Masi, S. [2016] Int. J. Mod. Phys. D 25(11), 1640012.

Di Valentino, E. et al. [2016] arXiv:1612.00021.

Essinger-Hileman, T., Kusaka, A. et al. [2016] Rev. Sci. Instrum. 87(9), 094503.

Gandilo, N. et al. [2014] Proc. SPIE 9145, 91452U.

Gerbino, M. et al. [2016] J. Cosmol. Astropart. Phys. 1607(07), 044.

Gluscevic, V. \& Kamionkowski, M. [2010] Phys. Rev. D 81, 12.

Gruppuso, A. et al. [2016] J. Cosmol. Astropart. Phys. 1606 (06), 001.

Gubitosi, G. et al. [2015] J. Cosmol. Astropart. Phys. 2014 (12), 020, astro-ph/1410.1799.

Houde, M. et al. [2001] Publ. Astron. Soc. Pac. 113, 622.

Hu, W. et al. [2003] Phys. Rev. D 67, 043004.

Johnson, B. R. et al. [2015] J. Astron. Instrum. 04, 1550007.

Jones, W. C. et al. [2007] A\&A 470, 771.

Kaufman, J. P. et al. [2014a] Phys. Rev. D 89, 062006.

Kaufman, J. P. et al. [2014b] MNRAS 455(2), 1981.
Keating, B. et al. [2013] ApJL 762, L23.

Koopman, B. et al. [2016] Proc. SPIE 9914, 99142T.

Kosowsky, A. \& Loeb, A. [1996] ApJ 469, 1.

Lamarre, J. M. [2016] Appl. Opt. 25, 6.

Lewis, A. et al. [2002] Phys. Rev. D 66, 103511.

Louis, T., Grace, E., Hasselfield, M. et al. [2016], astro-ph/ 1610.02360 .

MacTavish, C. J. et al. [2008] ApJ 689, 2.

Madhavacheril, M. et al. [2015] Phys. Rev. Lett. 114(15), 151302.

Miller, N. J. et al. [2009] Phys. Rev. D 79, 103002.

Miller, N. J. et al. [2016] ApJ 818, 151.

O'Dea, D. et al. [2007] MNRAS 376, 17671783.

Pagano, L. et al. [2009] Phys. Rev. D 80, 043522.

Perotto, L. et al. [2006] J. Cosmol. Astropart. Phys. 0610, 013.

Pisano, G. et al. [2014] Proc. SPIE 9153, 915317.

Planck Collaboration [2014a] A\&A 571, A16.

Planck Collaboration [2014b] A\&A 571, A15.

Planck Collaboration [2014c] A\&A 571, A17.

Planck Collaboration [2016a] A\&A 594, A13.

Planck Collaboration [2016b] $A \& A$ 594, A11

Planck Collaboration [2016c] $A \mathscr{E} A$ 594, A8.

Planck Collaboration [2016d] $A \& A$ 594, A15.

Planck Collaboration [2016e] A\&A 596, A102.

Planck Collaboration [2016f] A\&A 586, A133.

Planck Collaboration [2016g] $A \mathscr{G} A$ 596, A110.

Planck Collaboration [2016h] A\&A 594, A19.

Planck Collaboration [2016i] $A \mathscr{E} A$ 594, A8.

Pogosian, L. et al. [2012] astro-ph/1210.0308.

Rosset, C. et al. [2010] A\&A $\mathbf{5 2 0}, \mathrm{A} 13$.

Sherwin, B. et al. [2016], astro-ph/1611.09753.

Shimon, M. et al. [2008] Phys. Rev. D 77, 083003.

Simon, S. M. et al. [2016] J. Low. Temp. Phys. 184, 534.

Simons Observatory Collaboration [2016], https://simonsobservatory.org/.

The LSPE Collaboration [2012] Proc. SPIE 8846, 84467A.

The POLARBEAR Collaboration [2014] ApJ 794, 171.

Thornton, R. J. et al. [2016] ApJS 227, A21.

van Engelen, A. et al. [2016] ApJ 808, 7.

Weiland, J. L. et al. [2011] ApJS 192, 19.

Xia, J.-Q. et al. [2010] Phys. Lett. B 687(2), 129.

Yadav, A. P. S. et al. [2012] Phys. Rev. D 86, 083002. 